\title{
Early Morphologic Alterations in Renal Artery Wall and Renal Nerves in Response to Catheter-Based Renal Denervation Procedure in Sheep: Difference Between Single-Point and Multiple-Point Ablation Catheters
}

\author{
M. TÁBORSKÝ ${ }^{1}$, D. RICHTER ${ }^{1}$, Z. TONAR ${ }^{2}$, T. KUBÍKOVÁ ${ }^{2}$, A. HERMAN $^{3}$, \\ J. PEREGRIN ${ }^{3}$, L. ČERVENKOVÁ ${ }^{3}$, Z. HUSKOVÁ ${ }^{3}$, L. KOPKAN $^{3}$ \\ ${ }^{1}$ Department of Internal Medicine I - Cardiology, University Hospital Olomouc and Palacký \\ University, Olomouc, Czech Republic, ${ }^{2}$ Department of Histology and Embryology and Biomedical \\ Center, Faculty of Medicine in Pilsen, Charles University, Pilsen, Czech Republic, ${ }^{3}$ Center for \\ Experimental Medicine, Institute for Clinical and Experimental Medicine, Prague, Czech Republic
}

Received October 10, 2016

Accepted February 2, 2017

On-line April 12, 2017

\section{Summary}

Renal sympathetic hyperactivity is critically involved in hypertension pathophysiology; renal denervation (RDN) presents a novel strategy for treatment of resistant hypertension cases. This study assessed effects of two RDN systems to detect acute intravascular, vascular and peri-vascular changes in the renal artery, and renal nerve alterations, in the sheep. The procedures using a single-point or multi-point ablation catheters, Symplicity Flex $^{\top \mathrm{M}}$, Medtronic versus EnligHTN ${ }^{\top \mathrm{M}}$, St. Jude Medical were compared; the intact contralateral kidneys served as controls. Histopathological and immunohistochemical assessments were performed $48 \mathrm{~h}$ after RDN procedures; the kidney and suprarenal gland morphology was also evaluated. Special staining methods were applied for histologic analysis, to adequately score the injury of renal artery and adjacent renal nerves. These were more pronounced in the animals treated with the multi-point compared with the single-point catheter. However, neither RDN procedure led to complete renal nerve ablation. Forty-eight hours after the procedure no significant changes in plasma and renal tissue catecholamines were detected. The morphologic changes elicited by application of both RDN systems appeared to be dependent on individual anatomical variability of renal nerves in the sheep. Similar variability in humans may limit the therapeutic effectiveness of RDN procedures used in patients with resistant hypertension.

\section{Key words}

Renal denervation • Resistant hypertension • Experimental model

\section{Corresponding author}

M. Táborský, Department of Internal Medicine I - Cardiology, University Hospital Olomouc and Palacký University, I. P. Pavlova 6, 77520 Olomouc, Czech Republic. E-mail: milos.taborsky@fnol.cz

\section{Introduction}

Renal sympathetic innervation is involved not only in the regulation of renal function, circulating fluid volume and homeostasis of ions, but also in the regulation of blood pressure (Johns et al. 2011). Activation of efferent renal sympathetic nerves leads to water retention, sodium reabsorption and reduction of renal blood flow, as well as activation of the reninangiotensin-aldosterone system (DiBona 2000). Although the mechanisms that link these conditions in resistant hypertension are not fully elucidated, enhanced sympathetic nervous system activity is considered as a major contributing factor (Rippy et al. 2011, Tsioufis et al. 2011). Therefore catheter-based renal denervation (RDN) has been applied as a potential cost-effective approach for patients with resistant hypertension unresponsive to standard antihypertensive therapy (Rippy 
et al. 2011). Although this method has been very widely applied over the last five years and claimed to be effective for blood pressure (BP) reduction in select patients (Bhatt et al. 2014, Mahfoud et al. 2013), satisfactory safety and efficacy data are still not available.

The evidence from preclinical experimental studies related to the safety and effectiveness of different radiofrequency-based RDN systems is limited. A singlepoint catheter system (the Symplicity Flex from Medtronic) has been used extensively in pig, sheep and human studies (Rippy et al. 2011, Bhatt et al. 2014, Mahfoud et al. 2013, Booth et al. 2015, Sakakura et al. 2015). The originally promising results of the Symplicity HTN-1 and 2 studies have been recently challenged by the early-terminated Symplicity HTN-3 study, which proposed more rigorous endpoints than did the previous studies (Mahfoud et al. 2013). Furthermore, the preclinical animal studies provided only inconclusive data regarding effectiveness of the denervation procedures. Some recent studies also address the issue of the reinnervation of the kidney within months after RDN (Booth et al. 2015, Sakakura et al. 2015).

An innovative multi-ablation device, the EnligHTN $^{\mathrm{TM}}$ multi-electrode RNA catheter from St. Jude Medical has been tested in pig, dog and also in humans (Worthley et al. 2013, Henegar et al. 2014, Gan et al. 2015, Mahfoud et al. 2016). This multi-point ablation catheter operates at multiple depth levels of the renal artery at the same time. Thus the adjacent nerve bundle is supposed to be more affected by the radio-frequency energy applied; on the other hand, several locations of the vascular wall within this single cross section are also affected, which could result in severe damage of the renal artery. No data have been provided so far to compare the morphological effects of the one-point and multi-point systems in the acute phase.

In the context of the above evidence the main goal of this study was to assess the acute morphologic effects and thereby evaluate to some extent the safety of two renal denervation systems using a single-point or multi-point ablation catheters in the sheep. The safety was verified by angiography and the evaluation of the kidney and the suprarenal gland morphology. The effectiveness of either RDN procedure was assessed by comparing histopathological and immunohistochemical features of intra-vascular, vascular and peri-vascular structures between the renal artery subjected to RDN and the contralateral renal artery. In addition, the levels of catecholamines were determined in the intact and postRDN kidney.

\section{Methods}

The study performed in sheep (total $n=12$; body weight $35-48 \mathrm{~kg}$ ) was approved by the Institute for Clinical and Experimental Medicine Animal Care and Use Committee in accordance with the regulations accepted in the Czech Republic and guidelines from Directive 2010/63/EU of the European Parliament. The sheep model can be regarded as optimal for $\mathrm{RDN}$ in many respects: the adult animal is large and shows minimal body weight gain over time, which is of obvious advantage with long-term experiments. In consequence, the results of acute and chronic studies can be readily compared over prolonged period of time. Last but not least, the commercially available ablation catheters fit the diameter of the renal artery in the sheep.

\section{Renal artery angiography and RDN procedure}

The sheep were randomly assigned into two mixed-sex groups, each containing six animals. Anesthesia was induced with combination of propofol (10 mg/kg; Fresenius Kabi, Bad Homburg, Germany) and fentanyl (3 mg/kg; Torrex, Chiesi, Wien, Austria) given intravenously. After endotracheal intubation, anesthesia was maintained with $0.5 \%$ to $2.0 \%$ isoflurane (Isofluran Rhodia, Nicholas Piramar, Mumbai, India) in $\mathrm{O}_{2} /$ air mixture. The right femoral artery incision was performed as the access point for the catheterization, a sheath was introduced into the exposed artery and heparin (3000 IU) was administered. After that a pigtail catheter was inserted through the sheath into the abdominal aorta to reach the area where the renal arteries originate from the aorta. Angiography was performed using a standard contrast agent (Telebrix 35, Guerbet BP, Roissy CdG Cedex, France) injection to assess the above-defined area before application of the RDN procedure. The guiding catheter was inserted into the right renal artery and then the appropriate ablation catheter approved for the clinical use (Symplicity Flex ${ }^{\mathrm{TM}}$, Medtronic, Inc., Santa Rosa, CA, USA or EnligHTN ${ }^{\mathrm{TM}}$ Basket, St. Jude Medical, Inc., Saint Paul, MN, USA), was connected to a generator and RDN procedure was performed. Both RDN systems are fully automatic with predefined limits. The Medtronic generator utilizes an algorithm for the delivery within $120 \mathrm{~s}$ of radiofrequency energy (4-8 W) with maximal 
temperature limit $75^{\circ} \mathrm{C}$. The EnligHTN generator employs $60 \mathrm{~s}$ cycle of radiofrequency energy of 3-6 W for each of four electrodes, with maximal temperature limit $70^{\circ} \mathrm{C}$. When $\mathrm{RDN}$ procedure was completed, the ablation catheter was removed, and angiography of both arteries was repeated. After the experiments the catheter, wire and sheath were withdrawn, the femoral wound was closed and sheep were monitored till full recovery from anesthesia. For analgesia butorphanol $0.2-0.4 \mathrm{mg} / \mathrm{kg}$ s.c. (Torbugesic, Zoetis Manufacturing and Reserch Spain S.L., Vall de Bianya, Spain) was administered when needed.

Forty-eight hours post-RDN the animals were anesthetized and prepared (by the same procedure as described above) for another angiography of both renal arteries via left femoral artery. Then the abdomen was opened for gross examination of the kidneys, the tissue samples of the both intact and intervened kidney were taken and immediately frozen in liquid nitrogen, for later catecholamine assays. After that the animals were euthanized by an anesthetic overdose (thiopental, VUAB Brno, Czech Republic) together with $\mathrm{KCl}$, then the aorta was clamped proximally and the kidneys were flushed with $10 \%$ buffered formalin via an introduced angiography catheter. The whole block (the abdominal aorta with attached renal arteries and kidneys) was excised for histological examination.

\section{Histological processing}

From each animal under study, tissue samples were removed for histological assessment of renal arteries and the accompanying nerves (8-9 samples per each renal artery that were processed in series of transversal sections), the kidneys ( 8 samples to include all anatomical zones), and the suprarenal gland (1-2 samples to include each anatomical part). The extensive sampling scheme was identical for organs subjected to RDN and for the intact contralateral organs.

Seven staining procedures including four immunohistochemical methods were applied and combined to reveal the most important microscopic features of the vascular wall, sympathetic peripheral nerves, kidney and suprarenal gland morphology: Hematoxylin-eosin; Verhoeff's hematoxylin and green trichrome; Mallory trichrome; Orcein (Tanzer's orcein, Bowley Biochemical Inc., Danvers, MA, USA); the Monoclonal Mouse Anti-Human Smooth Muscle Actin Antibody, Clone 1A4, dilution 1:100, pretreatment
Target Retrieval Solution, $\mathrm{pH} 9.0,96{ }^{\circ} \mathrm{C}, 20 \mathrm{~min}$; the Monoclonal Mouse Anti-Human Neurofilament Protein Antibody, Clone 2F11, dilution 1:75, pretreatment Target Retrieval Solution, pH 6.0, $96^{\circ} \mathrm{C}, 20 \mathrm{~min}$; the Polyclonal Rabbit Anti-Tyrosine Hydroxylase, pretreatment Target Retrieval Solution, pH 9.0, $96^{\circ} \mathrm{C}, 20 \mathrm{~min}$; the Polyclonal Rabbit Anti-Human Von Willebrand Factor, solution $1: 200$, pretreatment $10 \mathrm{~min}$ in chilled acetone and Proteinase $\mathrm{K}$; the binding of the primary antibody was visualized using the peroxidase reaction and 3,3'-diaminobenzidine (these chemicals form DakoCytomation, Glostrup, Denmark).

Histological evaluation of the intact and post-RDN renal arteries, renal nerves, kidneys and suprarenal glands

The histological changes reflecting injuries found in the renal artery and in nerves surrounding the renal artery after $48 \mathrm{~h}$ post-RDN procedure, as well as their classification and scoring are given in Table 1. Typical examples of the renal artery injury are shown in Figure 1, and the injury of the renal nerves is presented in Figure 2. The scoring system reflected severity (or grade) of the damage to the artery wall and adjacent nerves, and the relative extent of these changes. After performing the scoring, the values calculated for each sample under study were listed and presented in Table 2.

Measurement of catecholamines in the renal tissue and plasma

The tissue samples were homogenized in phosphate buffer with protease inhibitors and ascorbic acid and centrifuged $\left(10000 \mathrm{G}, 10 \mathrm{~min}, 4^{\circ} \mathrm{C}\right)$; full blood samples with EDTA were centrifuged $(10000 \mathrm{G}, 10 \mathrm{~min}$, $4{ }^{\circ} \mathrm{C}$ ) and assayed by TriCat TM Elisa kit (IBL International, Hamburg, Germany).

\section{Statistical analysis}

Nonparametric statistics was used: the Wilcoxon matched pairs test was used to compare the results found in RDN intervened and intact organs of the same animal. The Mann-Whitney U-test was used to assess the differences between the results of intervention using two different types of catheters. The correlation between the parameters calculated from the scoring systems was evaluated using the Spearman correlation coefficient. These tests were used as available in the Statistica Base 11 package (StatSoft, Inc., Tulsa, OK, USA). 

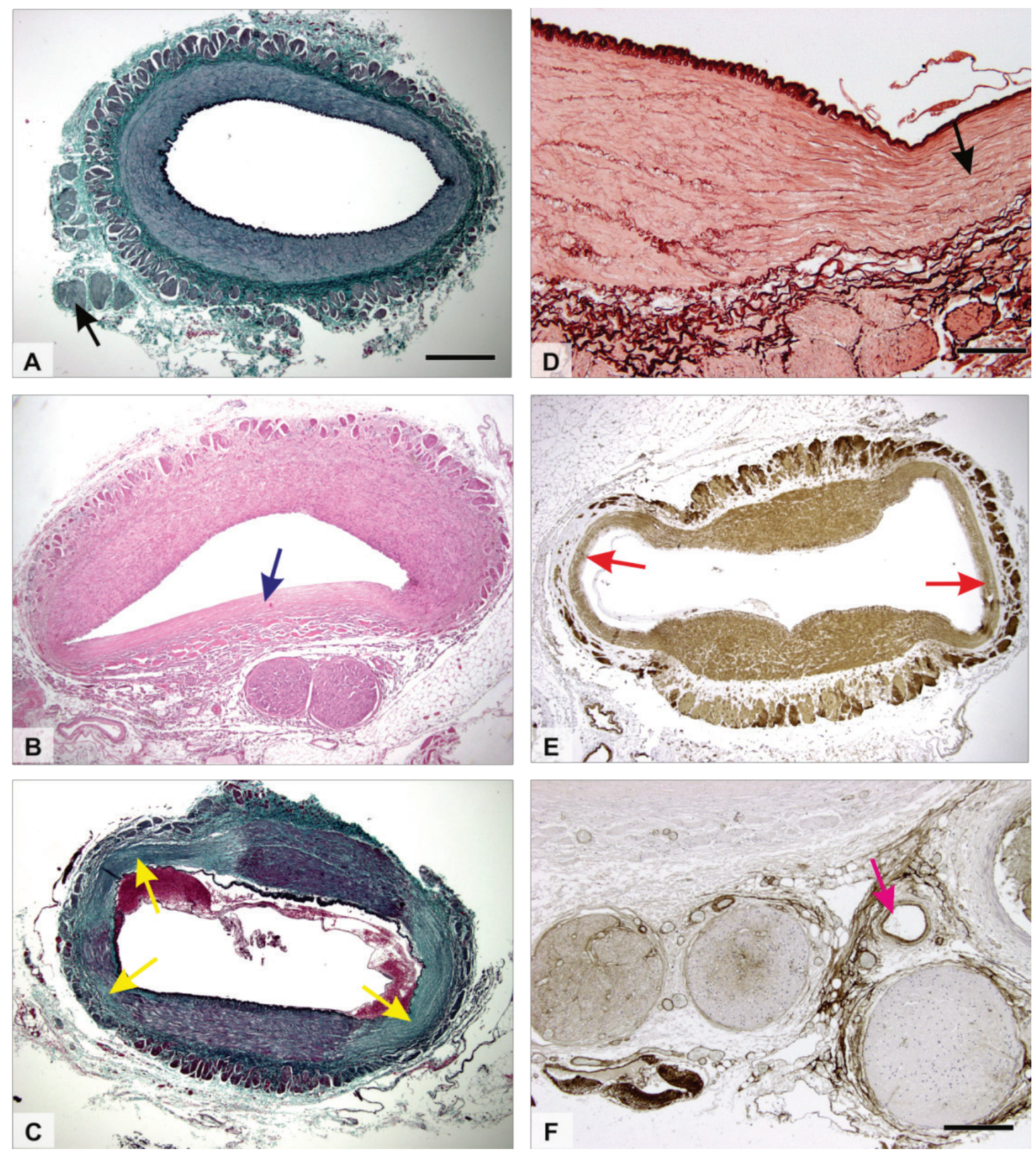

Fig. 1. Histological assessment of the RDN-induced injury of the renal artery in transverse histological sections, arranged using a semiquantitative scale. A) No histological signs of injury of either artery or nerves (black arrow). B) Eccentric focal damage and weakening of the wall (blue arrow). C) Collagen denaturation in three quadrants (yellow arrows). An intraluminal thrombus is visible. D) Damaged elastic membranes within the tunica media layer (black arrow). E) Loss of immunopositivity for alpha-smooth muscle actin in two quadrants (red arrows). F) Dilated or obliterated vasa vasorum in the adventitia of the artery (magenta arrow) close to the nerves. Verhoeff's hematoxylin and green trichrome staining $(\mathbf{A}, \mathbf{C})$, hematoxylin-eosin staining $(\mathbf{B})$, orcein staining (D, elastin stained dark brown), immunohistochemical detection of alpha-smooth muscle actin in vascular smooth muscle (E, dark brown positivity), immunohistochemical detection of von-Willebrand factor in endothelial cells (F, dark brown positivity). Scale bar $500 \mu \mathrm{m}(\mathrm{A}-\mathrm{C}, \mathrm{E})$, $100 \mu \mathrm{m}$ (D) and $200 \mu \mathrm{m}(\mathrm{F})$.

\section{Results}

Pre- and post-RDN angiography of the renal artery

The post-RDN angiography indicated that neither of RDN systems caused any serious alteration of renal blood flow in the intervened kidney confirming the safety of these procedures. No evidence of adverse effects, such as renal artery stenosis, thrombosis, perforation or spasm was apparent based on angiography immediately post-procedure or $48 \mathrm{~h}$ later, suggesting that no major renal vascular abnormality occurred after RDN procedure. 

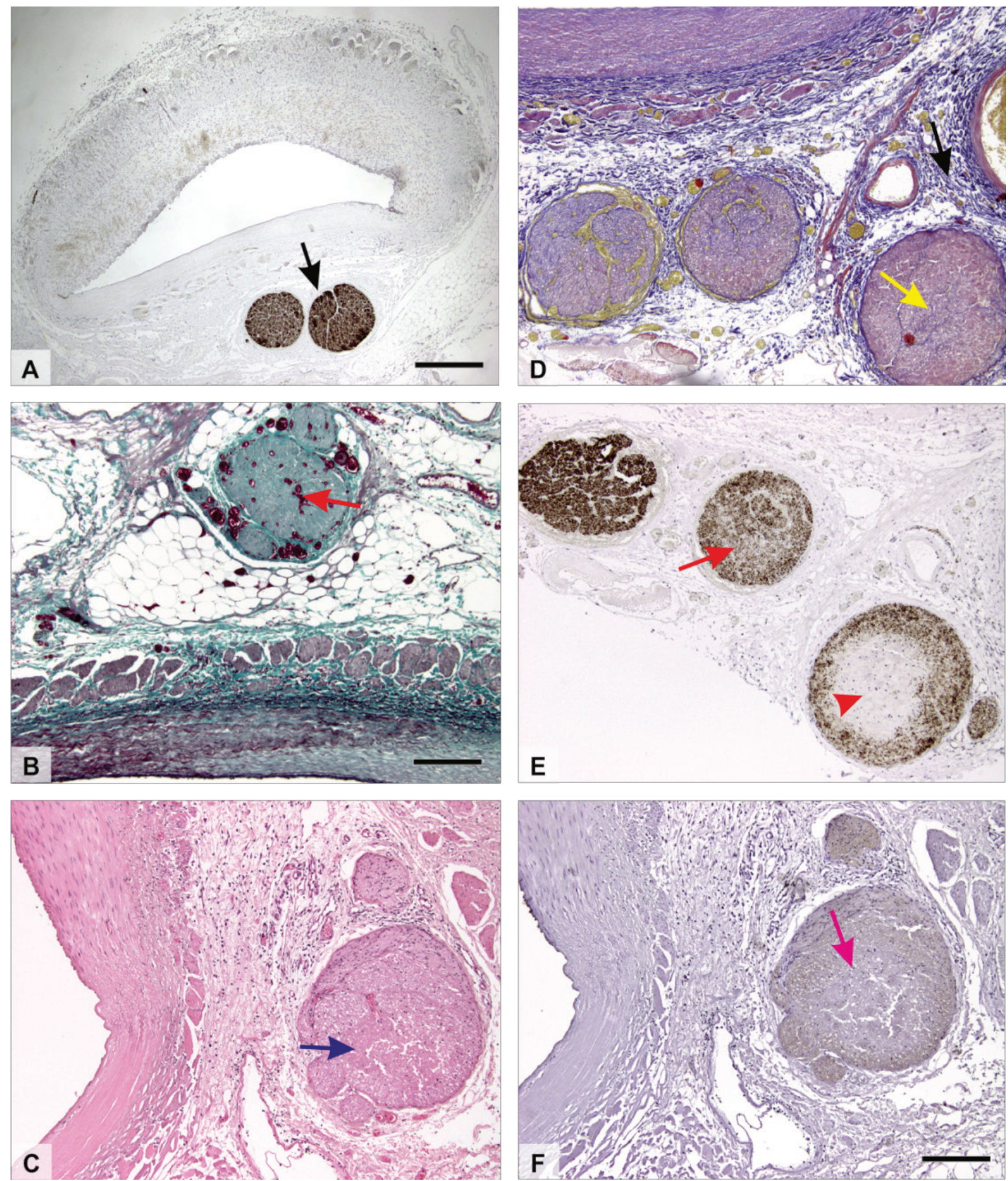

Fig. 2. Histological assessment of the RDN-induced injury of the nerves accompanying the renal artery in transverse histological sections, arranged using a semiquantitative scale. A) No histological signs of nerve injury (black arrow). B) Hyperemia of the nerve with dilated vasa nervorum (red arrow). C) Destructive fragmentation and complete loss of nuclei of Schwann glia cells (blue arrow). D) Loss of the fine structure of endoneurium connective tissue between the nerve fibres (yellow arrow). E) Moderate loss (red arrow) or complete loss (red arrowhead) of immunopositivity for neurofilament protein. F) Loss of immunopositivity for tyrosinehydroxylase (magenta arrow). Immunohistochemical detection of neurofilament protein (A, E, dark brown positivity). Verhoeff's hematoxylin and green trichrome staining (B), hematoxylin-eosin staining (C), Mallory trichrome staining (D), immunohistochemical detection of tyrosine-hydroxylase (F, dark brown positivity). Scale bar $500 \mu \mathrm{m}(\mathrm{A})$ and $200 \mu \mathrm{m}(\mathrm{B}-\mathrm{F})$.

Vascular and nerve injury status with and without RDN intervention

The scoring system showed that the sum, the maximum, and the mean vascular injury score were greater after intervention compared to the contralateral untreated renal artery in both groups of animals: those treated with the Symplicity Flex catheter and the EnligHTN catheter. However, in the former group the difference in nerve injury score did not reach significance when compared with the nerves surrounding the renal 
Table 1. Semiquantitative scale for assessing the injury of the renal artery and adjacent nerves in transverse histological sections. Points (1-4) were allocated per each arterial quadrant showing an injury of the artery or nerves in the segment under study. See Figures 1 and 2 for illustration of typical histological findings.

\begin{tabular}{ll}
\hline Points per each arterial segment & $\begin{array}{c}\text { Injury assessment of the post-RDN renal artery } \\
\text { Histological injury found in renal artery }\end{array}$ \\
\hline 0 & no histological signs of injury \\
$1,2,3,4$ & eccentric focal damage and weakening of the wall \\
$1,2,3,4$ & collagen denaturation \\
$1,2,3,4$ & damaged elastic membranes \\
$1,2,3,4$ & loss of immunopositivity for alpha-smooth muscle actin \\
$1,2,3,4$ & damage to the vasa vasorum \\
1 & presence of intraluminal thrombus \\
\hline
\end{tabular}

Injury assessment of the post-RDN peripheral nerves accompanying the renal artery Points per each nerve segment Histological injury found in nerves surrounding the renal artery

$\begin{array}{ll}0 & \text { no histological signs of injury } \\ 1,2,3,4 & \text { hyperemia of nerve } \\ 1,2,3,4 & \text { destructive fragmentation or complete loss of nuclei of Schwann cells } \\ \text { damage to the inner nerve structure: loss of neurilemma and structure of the } & \text { endoneurial connective tissue } \\ 1,2,3,4 & \text { loss of immunopositivity for neurofilament protein } \\ 1,2,3,4 & \text { loss of immunopositivity for tyrosine hydroxylase } \\ 1,2,3,4 & \end{array}$

Table 2. Parameters calculated from the semiquantitative scoring systems.

\begin{tabular}{ll}
\hline Parameter & Definition \\
\hline $\begin{array}{l}\text { Sum of the vascular injury score and } \\
\text { sum of the nerve injury score per } \\
\text { animal }\end{array}$ & $\begin{array}{l}\text { Calculated by summing up the points scored along all the } 3 \mathrm{~mm} \text {-long } \\
\text { segments of the renal artery (typically } 8 \text { segments). This was done for the } \\
\text { vaximum vascular injury score and } \\
\text { maximum nerve injury score }\end{array}$ \\
$\begin{array}{l}\text { The maximum value found among the segments under study in the given } \\
\text { animal and organ. }\end{array}$ \\
$\begin{array}{l}\text { Arithmetic mean value of the score results per one segment, calculated by } \\
\text { dividing the sum of the vascular injury score, or the sum of the nerve injury } \\
\text { per segment }\end{array}$ \\
$\begin{array}{l}\text { score, respectively, by the number of arterial segments in the given animal } \\
\text { and organ. }\end{array}$
\end{tabular}

artery without intervention. In contrast, the nerve injury score was significantly greater after intervention with the EnligHTN catheter compared to the nerves of the untreated renal artery (Fig. 3).

Vascular and nerve injury caused by the two types of the catheter

Compared with the impact of the Symplicity Flex catheter, RDN procedure using the EnligHTN catheter resulted in significantly greater injury to the renal artery (Fig. 3A) and also in a greater nerve injury score (Fig. 3B).

Typical histological findings showing the wall of renal arteries after RDN intervention are depicted in Figure 4. In the renal arteries treated with the Symplicity catheter, the lesions of the vascular wall were less extensive, often restricted to only one quadrant of the vascular section (Fig. 4A-C). In one animal, more 
quadrants were affected (Fig. 4D). In arteries treated with the EnligHTN catheter, the vascular lesions were more extensive, often affecting multiple quadrants in cross sections of several segments (Fig. 4E-H). Except for the circular layer of smooth muscle, all renal arteries examined in this sheep model had also an additional layer of longitudinal smooth muscle (Fig. 4).

Typical findings in the nerves accompanying the renal arteries are illustrated in Figure 5. In many sections, morphologically normal unaltered nerves were found, often running in the direction opposite to those in the damaged arterial quadrant (Figs 5A and 5E). All types of nerve injury as shown in Figures $5 \mathrm{~B}-\mathrm{D}$ and $5 \mathrm{~F}-\mathrm{H}$, and also in Figure 2, were more pronounced in the animals treated with the EnligHTN catheter when compared with those treated with the Symplicity catheter.

Histological findings in the kidneys and suprarenal glands after intervention

Figure 6 represents histological findings illustrating normal appearance of the kidneys and the suprarenal glands after either RDN intervention. No signs of ischemia, hemorrhage, necrosis, or increased leukocyte infiltration were found in the renal cortex (Figs 6A and $6 \mathrm{E}$ ) or medulla (Figs $6 \mathrm{~B}$ and $6 \mathrm{~F}$ ). Both neurofilament protein-positive as well as tyrosine-hydroxylase-positive nerve fibres were found in samples of all tissue blocks from all kidneys under study (Figs 6C and 6G). In all samples of the suprarenal gland, a well-defined tyrosinehydroxylase positive medulla was seen (Figs 6D and 6H); there was no difference between individual experimental groups.

Plasma and kidney catecholamine levels in anesthetized sheep

Compared to basal dopamine $(0.73 \pm 0.49 \mathrm{ng} / \mathrm{ml})$, norepinephrine $(1.70 \pm 0.86 \mathrm{ng} / \mathrm{ml})$, and epinephrine $(1.41 \pm 0.67 \mathrm{ng} / \mathrm{ml})$ levels in plasma of anesthetized sheep, RDN did not lead to any appreciable change in catecholamines immediately after procedure and also after $48 \mathrm{~h}$. Compared to the intact kidney, norepinephrine levels in the post-RDN kidney tended to be slightly higher (NS) in the RDN-treated kidney after $48 \mathrm{~h}$ (Symplicity Flex $10.7 \pm 3.6$ vs. $5.8 \pm 2.8 \mathrm{~g} / \mathrm{g}$ tissue; EnligHTN Basket $15.9 \pm 4.6$ vs. $8.6 \pm 2.2 \mathrm{~g} / \mathrm{g}$ tissue; respectively). A similar trend was seen in epinephrine levels (Symplicity Flex $14.8 \pm 6.7$ vs. $7.1 \pm 5.2 \mathrm{~g} / \mathrm{g}$ tissue; EnligHTN Basket $17.6 \pm 5.1$ vs. $10.0 \pm 2.1 \mathrm{~g} / \mathrm{g}$ tissue; respectively).

\section{A Sum of Vascular injury Score per Animal}

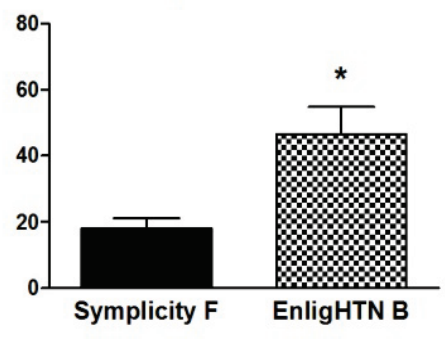

\section{B Sum of Renal Nerve injury Score per Animal}

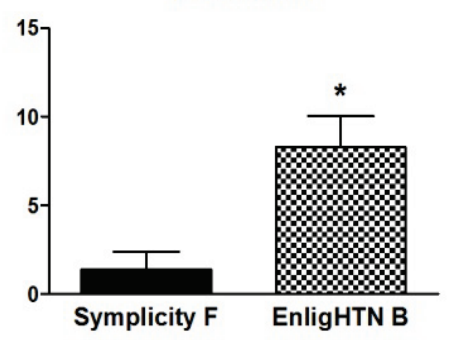

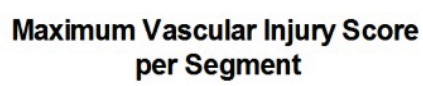

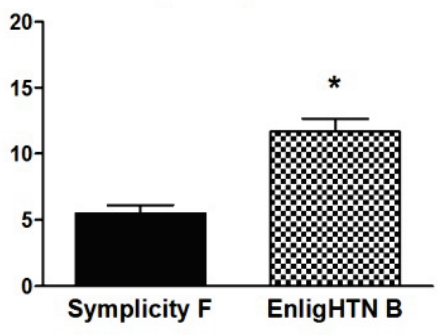

Maximum Renal Nerve Injury Score
per Segment

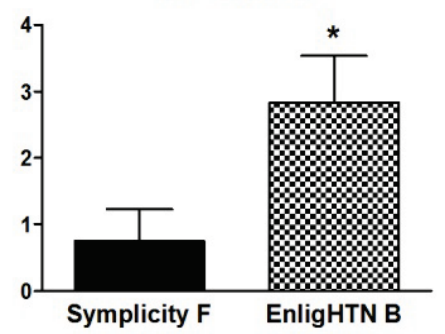

Mean Vascular Injury Score per Segment

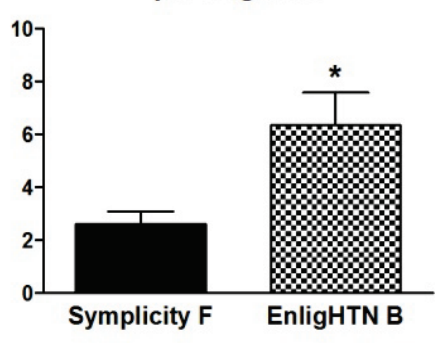

Mean Renal Nerve Injury Score
per Segment

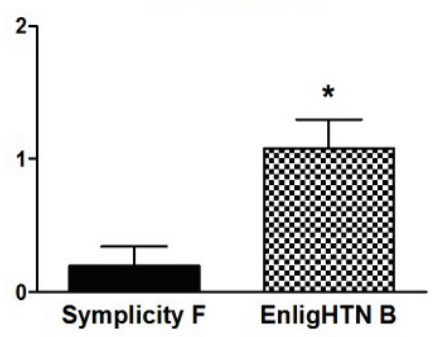

Fig. 3. Comparison of the the RDN-induced renal artery (A) and renal nerve (B) injury scores for single-point and multiple-ablation catheters. A) The sum, the maximum and the mean injury to the renal artery treated with the Symplicity Flex catheter or EnligHTN catheter. B) The sum, the maximum and the mean injury to the renal nerve treated with the Symplicity Flex catheter or EnligHTN catheter. Data are displayed as the mean \pm SEM, differences significant at $* p<0.05$. 

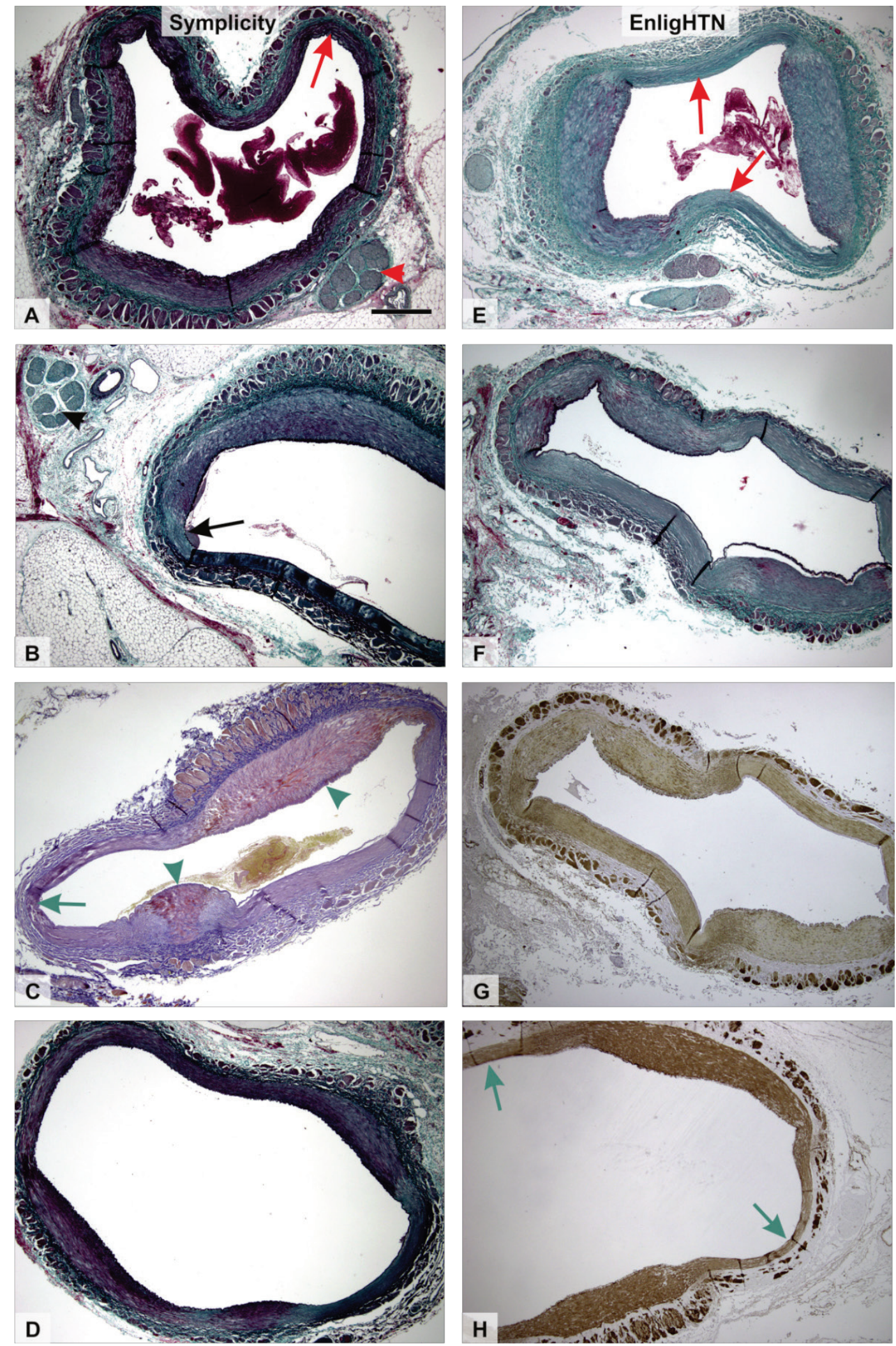

Fig. 4. Examples of histological signs of arterial injury in the animals after intervention using the Symplicity Flex catheter (left) or EnligHTN catheter (right). In the renal arteries treated with the Symplicity catheter, the lesions of vascular wall were less extensive, often affecting only one quadrant of the vascular section (A, B, arrrows). The accompanying nerves were often normal (A, B, arrowheads). Occasionally, the weakening of the wall and collagen denaturation with smooth muscle loss (C, arrow) was accompanied by adjacent edema of the tunica media layer (C, arrowheads). In one animal, more quadrants were affected (D). In the arteries treated with the EnligHTN catheter, the vascular lesions were more extensive, often affecting multiple quadrants in the cross sections of several segments (E-H, arrows in $\mathbf{E}, \mathbf{H})$. Verhoeff's iron hematoxylin and green trichrome stain (A, B, E-F), Mallory trichrome (C), immunohistochemical detection of alpha-smooth muscle actin (G-H, dark brown positivity). Scale bar $500 \mu \mathrm{m}(\mathrm{A}-\mathrm{H})$. 

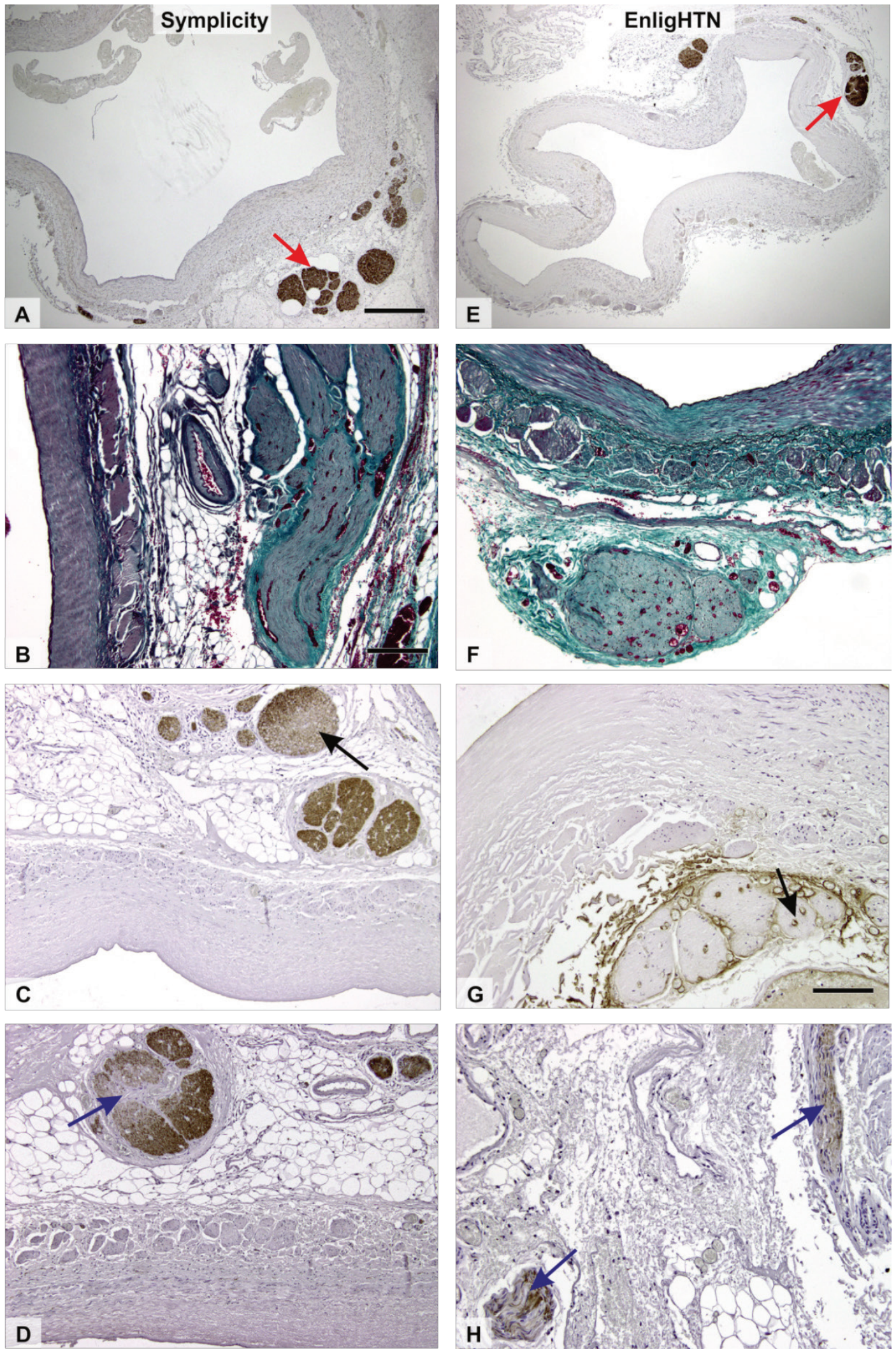

Fig. 5. Examples of histological signs of nerves damage in the animals after intervention using the Symplicity Flex catheter (left) and EnligHTN catheter (right). In many sections, adjacent to the renal artery, morphologically and immunohistochemically normal nerves were found ( $\mathbf{A}, \mathbf{E}$, red arrows). In both groups of animals, hyperemia of nerves without further damage to the nerve microstructure (B, F) was commonly seen. Despite complete immunohistochemical negativity (not shown here, see Fig. 2), some nerve bundles were partially damaged, showing focal loss of immunopositivity for neurofilament protein $(\mathbf{C})$ or tyrosine hydroxylase (D, H). In some nerve bundles, the vasa nervorum microvessels (G, black arrow) were either dilated or destroyed. Immunohistochemical detection of neurofilament protein $(\mathbf{A}, \mathbf{C}, \mathbf{E}$, dark brown positivity), immunohistochemical detection of tyrosine-hydroxylase (D, $\mathbf{H}$, dark brown positivity), Verhoeff's hematoxylin and green trichrome (B, F). Scale bar $500 \mu \mathrm{m}(A, E), 200 \mu \mathrm{m}(B-D, F)$ and $100 \mu \mathrm{m}$. 

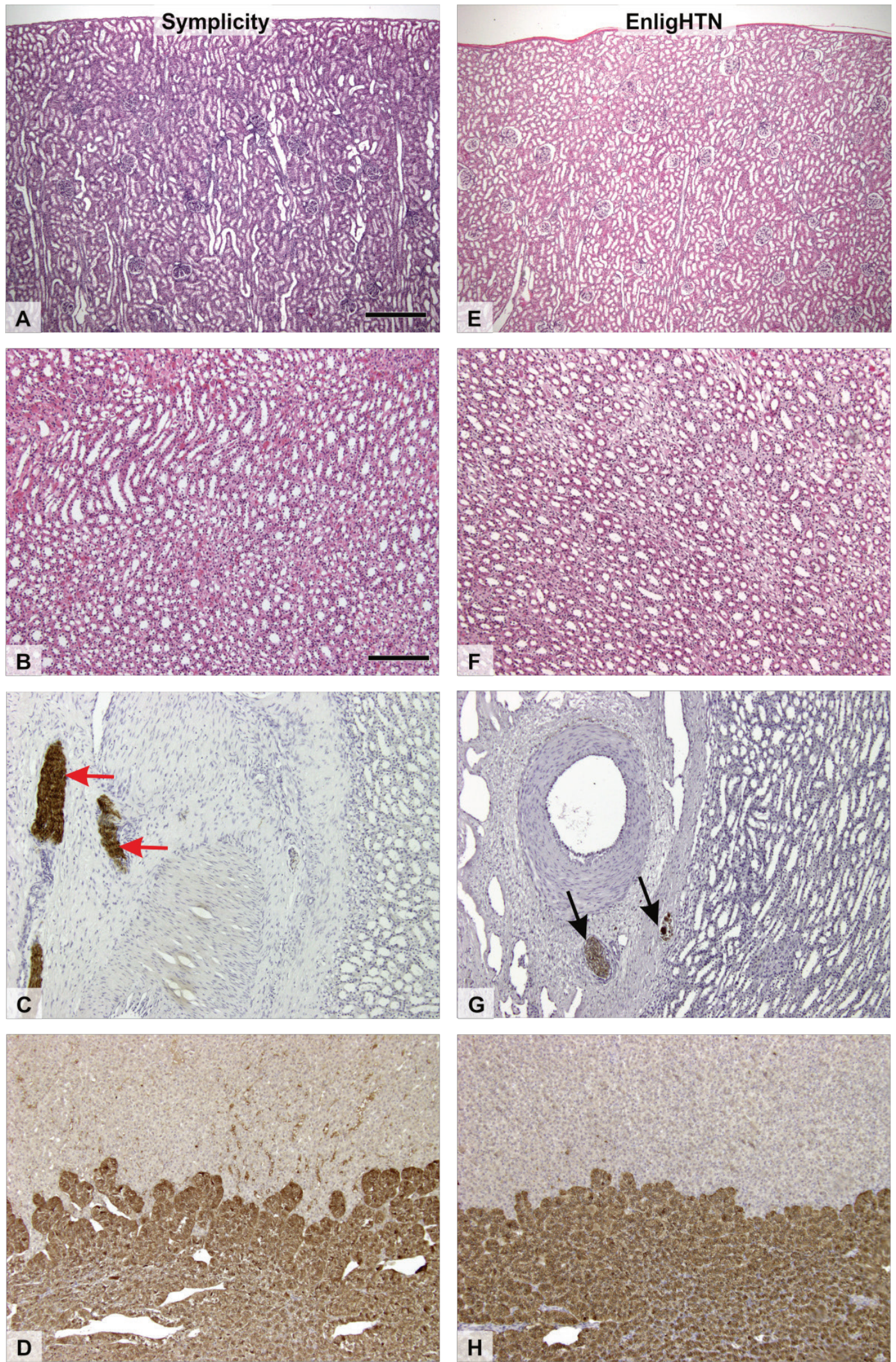

Fig. 6. Histological findings illustrating normal appearance of the kidneys and the suprarenal glands after intervention using the Symplicity Flex catheter (left) and EnligHTN catheter (right). Both kidney cortex (A, E) and medulla (B, F) showed no damage, necrosis, or inflammatory infiltration. In both groups of animals, nerves positive for immunohistochemical detection of neurofilament protein (C) and tyrosin hydroxylase (G) were found. The medulla of the suprarenal glands was positive for tyrosine-hydroxylase in both groups $(\mathbf{D}, \mathbf{H})$. Hematoxylin-eosin (A, B, E, F), immunohistochemical detection of neurofilament protein (C, dark brown positivity), immunohistochemical detection of tyrosine-hydroxylase (G-H, dark brown positivity). Scale bar $500 \mu \mathrm{m}(\mathrm{A}, \mathrm{E})$ and $200 \mu \mathrm{m}(\mathrm{B}-\mathrm{D}, \mathrm{F}-\mathrm{H})$. 


\section{Discussion}

In order to extend the knowledge obtained in earlier pre-clinical studies (Booth et al. 2015, Sakakura et al. 2015, Gan et al. 2015, Mahfoud et al. 2016), we aimed to examine the effects of the single-point and multi-point ablation catheters at $48 \mathrm{~h}$ post-RDN procedure. This early phase post-RDN has not yet been investigated. We confirmed that there was no noticeable alteration within the renal vascular bed (e.g. due to renal artery stenosis or occlusion by angiography), similarly so in the case of post-RDN and control kidneys. Furthermore, histological evaluation of the kidney and suprarenal gland tissue morphology did not reveal any significant changes in the parenchyma, such as ischemia or necrosis. Thus, our first conclusion is that in the sheep model neither RDN variant causes noticeable vascular complications, at least not within the first $48 \mathrm{~h}$.

Another focus in this study was histopathological and immunohistochemical evaluation of the possible acute intravascular, vascular and peri-vascular changes induced by exposure to radiofrequency energy, in comparison with the features of the renal artery and nerves of the contralateral kidney. The main finding was that the RDN procedure by multi-point ablation catheter led to significantly higher occurrence of vascular and peri-vascular damage, most likely due to superior efficacy of this procedure. Nevertheless, neither of the RDN systems achieved complete damage of the targeted renal nervous plexuses or significantly reduced the level of catecholamines in the post-RDN kidney within $48 \mathrm{~h}$. Thus our second conclusion is that RDN procedures caused renal nerve damage that was only incomplete, most likely due to anatomical variability of the renal artery wall, and of the variable peri-arterial renal nerve distribution in the sheep.

Although we are aware of gross anatomical and morphological differences between the relevant structures in the sheep and humans, the sheep model studies appear to be of clinical relevance. Besides different anatomical localization of the left kidney in sheep, we also noticed differences in the thickness of tunica media of the renal artery. Histomorphometric analysis has shown that the thickness of renal artery in human is significantly related to age, and both the intima and media undergo age-related hyperplasia (Reddy et al. 2011). Another issue is that in humans the peri-arterial renal nerve distribution with respect to density, size, and distance from renal artery lumen varies along the renal artery length (Sakakura et al. 2014). We observed that in the sheep the distance of peri-arterial renal nerve from renal artery lumen varied from 1 to $3 \mathrm{~mm}$. Overall, such individual variabilities may obviously affect the efficiency of RDN procedure to target renal innervation, and this study provides additional evidence that the extent of nerve structure damage during RDN depends on the distance from the ablation site. This observation may help explain the limited antihypertensive effects of RDN and possibly may also be relevant to the process of the subsequent progressive renal nerve regeneration and reinnervation of the kidneys (Booth et al. 2015, Sakakura et al. 2015).

A comparison of the morphologic effects of using single-point and multi-point ablation catheters strongly suggests that increasing the number of ablation sites increases the probability to induce damage to renal nerves (Tzafriri et al. 2014, Tsioufis et al. 2015, Rosa et al. 2016). Admittedly, the evidence on the long-lasting superior effects of multi-electrode RDN system still remains limited (Tsioufis et al. 2015, Rosa et al. 2016). Moreover, optical coherence tomography studies performed directly after the RDN procedure have shown that compared to the Simplicity RDN, the multi-electrode variant may increase the occurrence of thrombus formation (Templin et al. 2013). Unfortunately, although this method can precisely depict the vascular response to RDN procedure, it does not allow assessing the renal nerve damage and thus predict the denervation effectiveness. In the present study, the anatomical variability of the distribution of renal nerves in the sheep did not allow to achieve a complete or major disruption of the renal nerve bundle. Therefore it can be assumed that the effectiveness of any RDN system is highly influenced by the specific anatomical distribution of renal nerves and also by tissue conductivity determining energy transition from the ablation site to the peri-arterial nerve plexus (Sakakura et al. 2014). Furthermore, the nervous plexuses surrounding the renal artery have a considerable anatomical inter-individual variability in terms of a different number of nerves, different spatial distribution of their branching and anastomoses, and a variable distance from the renal artery (Reddy et al. 2011). Therefore, histological scoring systems seem most suitable for assessing the impact of renal denervation (Tzafriri et al. 2014). Our modified scoring system used in this study was focused on detailed evaluation of the variable microscopic changes of renal artery wall and the nerve microstructure following acute denervation. 
Although we observed that the multi-point ablation procedure resulted in a greater damage to renal nerves compared to the single-point ablation, our histologic analysis of renal nerves plexuses indicated still incomplete RDN effectiveness of both systems.

Numerous studies confirmed the value of renal tissue norepinephrine level as an index of RDN effectiveness (Krum et al. 2009, Cohen-Mazor et al. 2014, Booth et al. 2015). Renal denervation has also been shown to reduce whole-body norepinephrine spillover dependent on reduced sympathetic nerve signaling. However, the relationship between norepinephrine levels and the degree of renal nerve damage has never been established. Our results confirm that renal catecholamine content is not an early sensitive marker of the efficiency of RDN procedure, at least not within the first $48 \mathrm{~h}$. Moreover, one should be aware that catecholamine levels must also be affected by anesthesia and the stress underlying preparatory surgery and the denervation procedure. It seems that an appreciable response of renal norepinephrine content cannot be observed until after one week after denervation (Krum et al. 2009, Booth et al. 2015). This suggests that alterations in the renal nerves after RDN procedure can progress over days or weeks. Further studies are still required to provide adequate knowledge of this issue.

An obvious limitation of this study was that experiments were performed in normotensive animals with unaltered basal renal sympathetic activity. This could be expected to reduce the catecholamine level response to RDN in our sheep. Secondly, the very design of the study implied observation of the earliest morphologic alterations in renal artery wall and renal nerves in response to RDN; these results do not throw light on the expected further progression of vascular and peri-vascular changes, including the development of local fibrosis, or on the extent of functional responses to altered renal sympathetic activity. Another limitation is that histological processing of the tissue samples could have caused endothelial damage, therefore the value of the scoring of intra-vascular changes could be described as uncertain.

In conclusion, the effectiveness of the two catheter-based RDN systems approved for the clinical use seems largely dependent on anatomical interindividual variability of renal artery wall and adjacent renal nerves. Both systems tested in the present study provided only incomplete renal nerve ablation in the sheep. This observation indicates that the extensive variability of renal nerve plexuses and renal artery wall structure may limit the wide-range use of RDN procedure in patients with resistant hypertension.

\section{Conflict of Interest}

There is no conflict of interest.

\section{Acknowledgements}

This work was supported by the Ministry of Health of the Czech Republic grant 15-34123A awarded to M.T. and A.H. We would like to acknowledge the technical help of Zuzana Simunkova, Iveta Mrazova, Lenka Cervenkova and Sona Kikerlova.

\section{References}

BHATT DL, KANDZARI DE, O'NEILL WW, D'AGOSTINO R, FLACK JM, KATZEN BT, LEON MB, LIU M, MAURI L, NEGOITA M, COHEN SA, OPARIL S, ROCHA-SINGH K, TOWNSEND RR, BAKRIS GL; SYMPLICITY HTN-3 INVESTIGATORS: A controlled trial of renal denervation for resistant hypertension. N Engl J Med 370: 1393-1401, 2014.

BOOTH LC, NISHI EE, YAO ST, RAMCHANDRA R, LAMBERT GW, SCHLAICH MP, MAY CN: Reinnervation of renal afferent and efferent nerves at 5.5 and 11 months after catheter-based radiofrequency renal denervation in sheep. Hypertension 65: 393-400, 2015.

COHEN-MAZOR M, MATHUR P, STANLEY JR, MENDELSOHN FO, LEE H, BAIRD R, ZANI BG, MARKHAM PM, ROCHA-SINGH K: Evaluation of renal nerve morphological changes and norepinephrine levels following treatment with novel bipolar radiofrequency delivery systems in a porcine model. J Hypertens $\mathbf{3 2}$ : 1678-1691, 2014.

DIBONA GF: Nervous kidney: interaction between renal sympathetic nerves and the renin-angiotensin system in the control of renal function. Hypertension 36: 1083-1088, 2000. 
GAN Q, QU XK, GONG KZ, GUAN SF, HAN WZ, DAI JJ, LI RG, ZHANG M, LIU H, XU YJ, ZHANG YJ, FANG WY: Efficacy and safety of a novel multi-electrode radiofrequency ablation catheter for renal sympathetic denervation in pig. $J$ Geriatr Cardiol 12: 618-625, 2015.

HENEGAR JR, ZHANG Y, RAMA RD, HATA C, HALL ME, HALL JE: Catheterbased radiorefrequency renal denervation lowers blood pressure in obese hypertensive dogs. Am J Hypertens 27: 1285-1292, 2014.

JOHNS EJ, KOPP UC, DIBONA GF: Neural control of renal function. Compr Physiol 1: 731-767, 2011.

KRUM H, SCHLAICH M, WHITBOURN R, SOBOTKA PA, SADOWSKI J, BARTUS K, KAPELAK B, WALTON A, SIEVERT H, THAMBAR S, ABRAHAM WT, ESLER M: Catheter-based renal sympathetic denervation for resistant hypertension: a multicentre safety and proof-of-principle cohort study. Lancet 373: 1275-1281, 2009.

MAHFOUD F, LÜSCHER TF, ANDERSSON B, BAUMGARTNER I, CIFKOVA R, DIMARIO C, DOEVENDANS P, FAGARD R, FAJADET J, KOMAJDA M, LEFÈVRE T, LOTAN C, SIEVERT H, VOLPE M, WIDIMSKY P, WIJNS W, WILLIAMS B, WINDECKER S, WITKOWSKI A, ZELLER T, BÖHM M; EUROPEAN SOCIETY OF CARDIOLOGY: Expert consensus document from the European Society of Cardiology on catheter-based renal denervation. Eur Heart J 34: 2149-2157, 2013.

MAHFOUD F, MOON LB, PIPENHAGEN CA, JENSEN JA, PATHAK A, PAPADEMETRIOU V, EWEN S, LINZ D, BÖHM M: Catheter-based radio-frequency renal nerve denervation lowers blood pressure in obese hypertensive swine model. J Hypertens 34: 1854-1862, 2016.

REDDY S, KUMAR P, PRASAD K: Histomorphometric and sympathetic innervation of the human renal artery: a cadaveric study. Urol Ann 3: 141-146, 2011.

RIPPY MK, ZARINS D, BARMAN NC, WU A, DUNCAN KL, ZARINS CK: Catheter-based renal sympathetic denervation: chronic preclinical evidence for renal artery safety. Clin Res Cardiol 100: 1095-1101, 2011.

ROSA J, WIDIMSKÝ P, WALDAUF P, LAMBERT L, ZELINKA T, TÁBORSKÝ M, BRANNY M, TOUŠEK P, PETRÁK O, ČURILA K, BEDNÁŘ F, HOLAJ R, ŠTRAUCH B, VÁCLAVÍK J, NYKL I, KRÁTKÁ Z, KOCIÁNOVÁ E, JIRAVSKÝ O, RAPPOVÁ G, INDRA T, WIDIMSKÝ J JR: Role of adding spironolactone and renal denervation in true resistant hypertension: one-year outcomes of randomized PRAGUE-15 study. Hypertension 67: 397-403, 2016.

SAKAKURA K, LADICH E, CHENG Q, OTSUKA F, YAHAGI K, FOWLER DR, KOLODGIE FD, VIRMANI R, JONER M: Anatomic assessment of sympathetic peri-arterial renal nerves in man. J Am Coll Cardiol 64: 635-643, 2014.

SAKAKURA K, TUNEV S, YAHAGI K, O'BRIEN AJ, LADICH E, KOLODGIE FD, MELDER RJ, JONER M, VIRMANI R: Comparison of histopathologic analysis following renal sympathetic denervation over multiple time points. Circ Cardiovasc Interv 8: e001813, 2015.

TEMPLIN C, JAGUSZEWSKI M, GHADRI JR, SUDANO I, GAEHWILER R, HELLERMANN JP, SCHOENENBERGER-BERZINS R, LANDMESSER U, ERNE P, NOLL G, LÜSCHER TF: Vascular lesions induced by renal nerve ablation as assessed by optical coherence tomography: pre- and post-procedural comparison with the Simplicity catheter system and the EnligHTN multi-electrode renal denervation catheter. Eur Heart J 34: 2141-2148, 2013.

TSIOUFIS C, KORDALIS A, FLESSAS D, ANASTASOPOULOS I, TSIACHRIS D, PAPADEMETRIOU V, STEFANADIS C: Pathophysiology of resistant hypertension: the role of sympathetic nervous system. Int J Hypertens 2011: 642416, 2011.

TSIOUFIS CP, PAPADEMETRIOU V, DIMITRIADIS KS, KASIAKOGIAS A, TSIACHRIS D, WORTHLEY MI, SINHAL AR, CHEW DP, MEREDITH IT, MALAIAPAN Y, THOMOPOULOS C, KALLIKAZAROS I, TOUSOULIS D, WORTHLEY SG: Catheter-based renal denervation for resistant hypertension: twenty-four month results of the EnligHTN I first-in-human study using a multi-electrode ablation system. Int J Cardiol 201: 345-350, 2015.

TZAFRIRI AR, MAHFOUD F, KEATING JH, MARKHAM PM, SPOGNARDI A, WONG G, FUIMAONO K, BÖHM M, EDELMAN ER: Innervation patterns may limit response to endovascular renal denervation. $J \mathrm{Am}$ Coll Cardiol 64: 1079-1087, 2014. 
WORTHLEY SG, TSIOUFIS CP, WORTHLEY MI, SINHAL A, CHEW DP, MEREDITH IT, MALAIAPAN Y, PAPADEMETRIOU V: Safety and efficacy of a multi-electrode renal sympathetic denervation system in resistant hypertension: the EnligHTN I trial. Eur Heart J 34: 2132-2140, 2013. 